Z Herz-Thorax- Gefäßchir 2008 22 DOI 10.1007/s00398-008-0660-2 Online publiziert: 24. Oktober 2008 ๑) Springer Medizin Verlag 2008
Zum Artikel „Stellenwert ärztlicher Leitlinien in der Arzthaftungsbegutachtung" von Günter Ollenschläger in Z Herz- ThoraxGefäßchir 2008 22: 000-000 kommentiert vom Managing Editor Prof. Hans-Reinhard Zerkowski

„-eitlinien - Entscheidungskorridore begründet auf best verfügbarer Evidenz" hat die DGTHG diesen Sektor ihres Internetauftritts überschrieben. Diese Leitlinien sollen, als evidenzbasierte Schlüsselempfehlungen, Entscheidungshilfen sein im Bestreben, jedem einzelnen Patienten - wissenschaftlich fundiert - die aktuell für seine Situation beste Behandlung zukommen zu lassen. Besondere Bedeutung haben hierbei Nationale Versorgungsleitlinien und diejenigen Leitlinien, die der Entwicklung durch die AMWF unterworfen sind und somit konsentierten Empfehlungen aller an der Versorgung beteiligten Fachdisziplinen entsprechen. Deren Verbreitung und Implementierung in den Behandlungsalltag sind erklärtes Ziel. Scherbaum et al. betonen im Deutschen Ärzteblatt [1], dass Leitlinien darauf abzielen, „, unter Berücksichtigung der vorhandenen Ressourcen die Qualität der Versorgung zu verbessern, gute klinische Praxis zu unterstützen und die Stellung des Patienten zu stärken“. Eine Überzeugung, die wir teilen.

Ein prominent platzierter Kommentar [2] zu einem Beschluss des 6. Zivilsenats des BGH, der da lautet, dass „Leitlinien [...] - im Gegensatz zu den Richtlinien

\title{
Kommentar zum Beitrag „Stellenwert ärztlicher Leitlinien in der Arzt- haftungsbegutachtung"
}

des Gemeinsamen Bundesausschusses nicht unbesehen mit dem zur Beurteilung eines Behandlungsfehlers gebotenen medizinischen Standard gleichgesetzt werden " könnten, trägt zur Verunsicherung und Behinderung des Umsetzens von Leitlinienprogrammen bei. Zudem sich hier anschließt: „Danach erteilt der BGH den Anhängern einer Leitlinieneuphorie eine deutliche Absage." Nun ist man innerhalb der Arbeit mit EbM meist eher "trocken rational" und muss oft euphorische Zukunftsphantasien auf den Boden der Realität holen, dennoch ist eine solche Verkürzung der Sicht problematisch. Auch hier liegt der Teufel im Detail - innerhalb der Justiz kann die heute sicher häufig begründete Sorge vor Überregulierung auch nicht zur Abschaffung aller Gesetze führen, um der Euphorie beim Versuch der Ordnung des Gemeinwesens einen Riegel vorzuschieben.

Da die wenigsten unter uns regelmäßig mit entsprechendem Wissenshintergrund BGH-Urteile und deren Kommentierungen im Urtext lesen, haben wir uns entschlossen, einen Artikel Günter Ollenschlägers in überarbeiteter Fassung nachzudrucken, der dazu angetan ist, den vermeintlichen Widerspruch zwischen höchstrichterlicher Rechtsprechung und Leitlinienumsetzung aufzulösen.

Prof. Dr. med. Hans-Reinhard Zerkowski Bahnhofstraße 25, 4125 Riehen, Schweiz

\author{
Literatur \\ 1. Scherbaum WA et al (2008) Diabetes-Leitlinien. \\ Gemeinsames Handeln aller Beteiligten. Dtsch \\ Ärztebl; 105(33):A 1734-1736 \\ 2. Wienke A (2008) Mitteilungen. In: Deutsche Gesell- \\ schaft für Chirurgie (3):267
}

